\title{
Population genetics of Aedes albopictus (Diptera: Culicidae) in its native range in Lao People's Democratic Republic
}

\author{
Maysa Tiemi Motoki ${ }^{1,2,3^{*}}$, Dina Madera Fonseca ${ }^{4}$, Elliott Frederic Miot ${ }^{1,5,6,7}$, Bruna Demari-Silva $^{8}$, \\ Phoutmany Thammavong ${ }^{1}$, Somsanith Chonephetsarath'1, Nothasine Phommavanh'1, Jeffrey Conrad Hertz ${ }^{9}$, \\ Pattamaporn Kittayapong ${ }^{10}$, Paul Trevor Brey ${ }^{1+}$ and Sebastien Marcombe ${ }^{1+}$
}

\begin{abstract}
Background: The Asian tiger mosquito, Aedes (Stegomyia) albopictus (Skuse) is an important worldwide invasive species and can be a locally important vector of chikungunya, dengue and, potentially, Zika. This species is native to Southeast Asia where populations thrive in both temperate and tropical climates. A better understanding of the population structure of Ae. albopictus in Lao PDR is very important in order to support the implementation of strategies for diseases prevention and vector control. In the present study, we investigated the genetic variability of Ae. albopictus across a north-south transect in Lao PDR.

Methods: We used variability in a 1337-bp fragment of the mitochondrial cytochrome c oxidase subunit 1 gene (cox1), to assess the population structure of Ae. albopictus in Lao PDR. For context, we also examined variability at the same genetic locus in samples of Ae. albopictus from Thailand, China, Taiwan, Japan, Singapore, Italy and the USA.

Results: We observed very high levels of genetic polymorphism with 46 novel haplotypes in Ae. albopictus from 9 localities in Lao PDR and Thailand populations. Significant differences were observed between the Luangnamtha population and other locations in Lao PDR. However, we found no evidence of isolation by distance. There was overall little genetic structure indicating ongoing and frequent gene flow among populations or a recent population expansion. Indeed, the neutrality test supported population expansion in Laotian Ae. albopictus and mismatch distribution analyses showed a lack of low frequency alleles, a pattern often seen in bottlenecked populations. When samples from Lao PDR were analyzed together with samples from Thailand, China, Taiwan, Japan, Singapore, Italy and the USA, phylogenetic network and Bayesian cluster analysis showed that most populations from tropical/subtropical regions are more genetically related to each other, than populations from temperate regions. Similarly, most populations from temperate regions are more genetically related to each other, than those from tropical/subtropical regions.

Conclusions: Aedes albopictus in Lao PDR are genetically related to populations from tropical/subtropical regions (i.e. Thailand, Singapore, and California and Texas in the USA). The extensive gene flow among locations in Lao PDR indicates that local control is undermined by repeated introductions from untreated sites.
\end{abstract}

Keywords: Aedes albopictus, cox1 gene, Genetic population, Lao PDR

\footnotetext{
*Correspondence: maysatm@gmail.com

${ }^{\dagger}$ Paul Trevor Brey and Sebastien Marcombe contributed equally to this

work

${ }^{1}$ Medical Entomology and Vector Borne Disease Unit, Institut Pasteur du

Laos, Vientiane, Lao People's Democratic Republic

Full list of author information is available at the end of the article
} 


\section{Background}

Dengue fever, the potentially deadly outcome of infection with a mosquito borne flavivirus (DENV, Flaviviridae, Flavivirus), is one of the most challenging public health problems in the Greater Mekong Subregion (GMS) composed of Cambodia, China, Myanmar, Thailand, Vietnam and Lao People's Democratic Republic (PDR) [1, 2]. From 2009 to 2012, dengue was reported in all provinces in Lao PDR, except for Phongsaly and Huaphanh provinces in northern region [3]. All four serotypes of dengue flaviviruses (DENV1-4) now circulate in rural and urban areas in Lao PDR [3-7]. In Lao PDR, an extensive dengue outbreak, mostly attributed to serotype 3 (DENV3) in 2013, caused 44,098 cases and 95 deaths [8,9]. Again in 2017, 9832 cases of dengue fever were reported in Lao PDR, including 14 deaths, with the most affected provinces being Vientiane Capital and Champasak [10]. Both Aedes (Stegomyia) aegypti (Linnaeus) and Aedes (Stegomyia) albopictus (Skuse, 1894) were suspected to have been involved in these epidemics $[11,12]$. However, still there is no study proving their vector status in the country.

Aedes albopictus, the Asian tiger mosquito, is thought to be native to Southeast Asia [13]. In recent decades, Ae. albopictus has spread throughout the world and is now found on all continents except Antarctica [14-16]; it is considered one of the most invasive and widespread mosquito species in the world $[14,17]$. Despite Ae. albopictus being considered a secondary vector of dengue and chikungunya (CHIKV, Togaviridae, Alphavirus) relative to Ae. aegypti [18], in some instances such as in central Africa, China and Mediterranean Europe [19-21] it can become the primary vector. Of note, several laboratory studies have shown that Ae. albopictus can be more competent at transmitting DENV and CHIKV than Ae. aegypti [22-24]. Furthermore, Ae. albopictus has been associated with the emergence of Zika virus from its native Africa, although this is still in early stages of investigation [25-27].

Although mosquito populations with different genetic makeup may differ in vector competence [28], there is currently no information about the population genetics of Ae. albopictus in Lao PDR. Information about genetic diversity and population structure can be a tool in the development of effective mosquito control programmes $[29,30]$. Therefore, we obtained samples of Ae. albopictus from eight provinces from the northwest, northern, central and southern regions of Lao PDR including the two most affected provinces, Vientiane Capital and Champasak, and sequenced a fragment of the cytochrome $c$ oxidase subunit 1 gene (cox1) mitochondrial (mt) DNA. First, we analyzed the genetic variability of samples from Lao PDR, then compared against other samples from China, Japan, Taiwan, Singapore, the USA, Italy [31] and
Thailand to check the genetic relationships among them. Our primary aim was to increase our understanding of the population structure of Ae. albopictus in Laos in order to develop better strategies for dengue prevention and vector control in Lao PDR.

\section{Methods \\ Mosquito collection and identification}

The collections were carried out in eight localities from the northwest [Bokeo (BK), Luangnamtha (LN) and Xayabouly (XB) Provinces], northern [Luang Prabang (LP) Province], central [Vientiane prefecture (VC), Borikhamxay (BK) and Khammuane (KM) Provinces] and southern [Champasak $(\mathrm{CH})$ Province] regions of Lao PDR (Fig. 1). Aedes albopictus larvae and pupae were collected between 2014 and 2016 from domestic containers (tanks and jars) and peri-domestic habitats (used tires, discarded containers, etc.), then carefully transferred into WhirlPak plastic bags (BioQuip, Rancho Dominguez, CA, USA) and sent to the insectaries in Vientiane for rearing (field generation, F0). Each mosquito population sample consisted of larvae and pupae collected from at least 10 breeding sites per locality to reduce the likelihood of re-sampling them. Female mosquitoes were then stored individually in a desiccated tube at $-80{ }^{\circ} \mathrm{C}$ until molecular analyses. All mosquitoes were morphologically identified as Ae. albopictus using available keys [32] and confirmed by comparison of cox 1 barcode region sequences available on GenBank.

\section{DNA extraction and sequencing}

Total genomic DNA was extracted from single whole mosquitoes using a NucleoSpin ${ }^{\circledR}$ Tissue kit (MachereyNagel, Duren, Germany) according to manufacturer's instructions. The fragment of mtDNA cytochrome $c$ oxidase subunit 1 ( $\operatorname{cox} 1$ ) gene was amplified using two sets of primers, 1454F (5'-GGT CAA CAA ATC ATA AAG ATA TTG G-3') and 2160R (5'-TAA ACT TCT GGA TGA CCA AAA AAT CA-3'); and 2027F (5'-CCC GTA TTA GCC GGA GCT AT-3') and 2886R (5'-ATG GGG AAA GAA GGA GTT CG-3'), following the polymerase chain reaction (PCR) protocol explicitly detailed in Zhong et al. [31]. Aliquots of the PCR products were visualized on $1.5 \%$ agarose gels and successful amplifications were purified using ExosapIT ${ }^{\circledR}$ (USB Co, Cleveland, $\mathrm{OH}, \mathrm{USA})$. All sequencing reactions were carried out in both directions using an ABI Big Dye Terminator Kit v.3.1 (Applied Biosystems, Warrington, UK) and analyzed on an ABI Prism 3500xL-Avant Genetic Analyzer (Applied Biosystems, Foster City, CA, USA) at the Institut Pasteur du Laos sequencing facilities in Vientiane. 


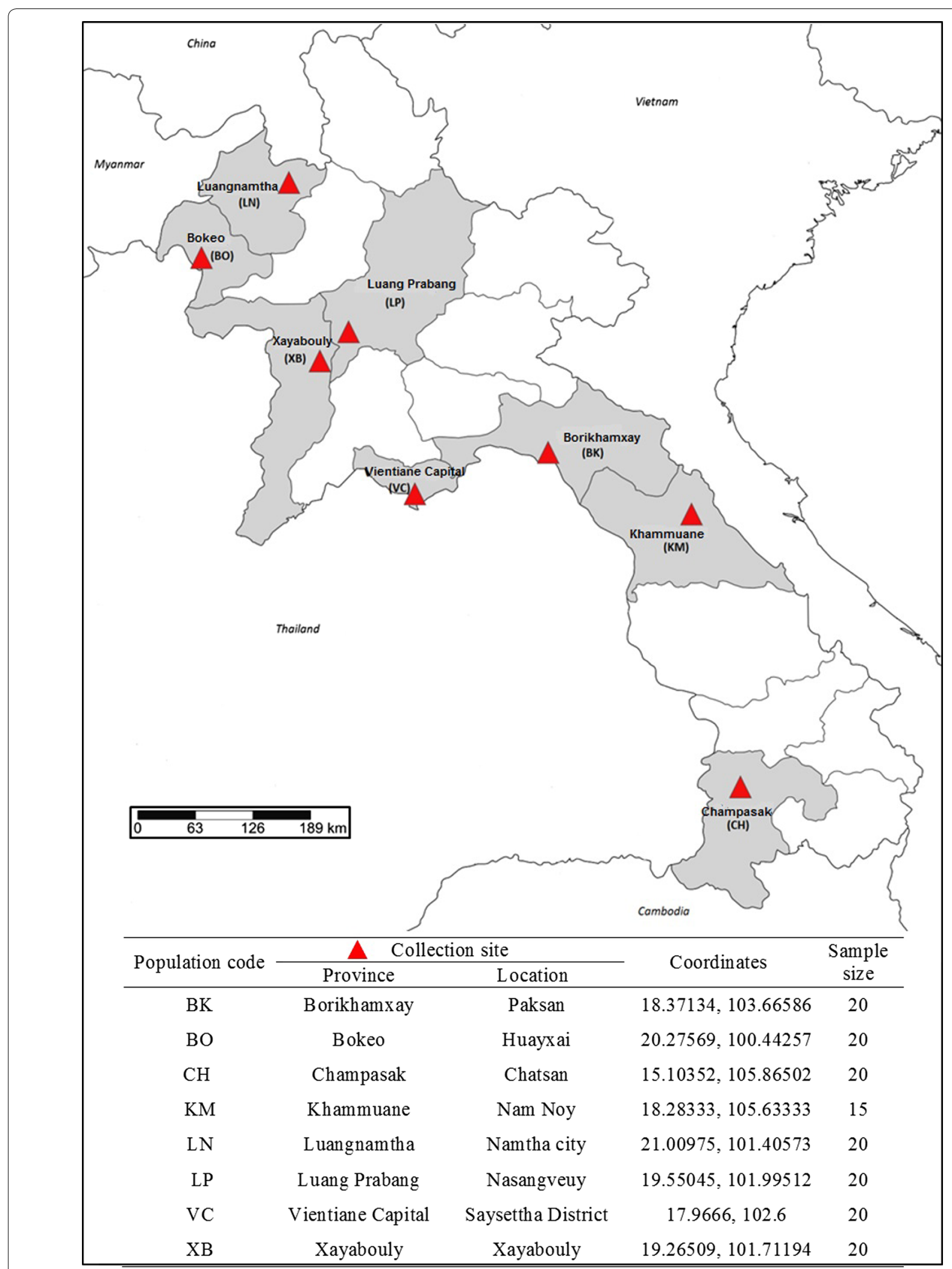

Fig. 1 Collection information of Aedes albopictus in Lao PDR 


\section{Data analyses}

The $\operatorname{cox} 1$ gene sequences were edited using Sequencher ${ }^{\circledR}$ version 5.4.6 (Gene Codes Corporation, Ann Arbor, MI, USA) and automatically aligned in Geneious v.9.1.6. [33].

The number of haplotypes $(\mathrm{H})$, haplotype diversity $(H d)$, nucleotide diversity $(\pi)$ and $(K)$ average of nucleotide differences within each site were generated using DnaSP v.5.0 [34]. The pairwise $F_{S T}$ was calculated to estimate population differentiation based on differences in haplotype frequencies, whereas Nei's $\mathrm{Nm}$ estimated gene flow is based on $G_{S T}$ [35] using Arlequin v.3.5 [36].

Analysis of molecular variance (AMOVA) was conducted to determine the distribution of genetic variation within and among populations using 1000 permutations implemented in Arlequin v.3.5 [36]. Additionally, a spatial analysis of molecular variance (SAMOVA) v.1.0 [37] was used to cluster the 1337-bp cox1 sequences into genetically and geographically homogeneous populations. SAMOVA generates $F$-statistics $\left(F_{S C}, F_{S T}, F_{C T}\right)$, using the AMOVA approach, into $K$ groups to maximize the between group variation. SAMOVA estimates were computed for $K=2-8$, with 1000 simulated annealing steps from each of 100 sets of initial starting conditions. Isolation by distance (IBD) was checked using a Mantel tests [38]. IBD was estimated in GenAlEx v.6.5 [39, 40] between the genetic and geographical distances with 10,000 permutations.

The hypothesis of strict neutrality among Ae. albopictus populations from Lao PDR was examined using the statistics $D$ [41] and Fu's $F_{S}$ [42], calculated using DnaSP v.5.0 [34]. The mismatch distribution (simulated in Arlequin v.3.5) was performed to distinguish between a smooth unimodal distribution and a multimodal or ragged distribution [43-45]. Statistically significant differences between observed and simulated distributions were evaluated with the sum of square deviations (SSD) to reject the hypothesis of demographic expansion [46].

To make broader comparisons among haplotypes from Lao PDR and other geographical regions, we analyzed samples from Thailand and downloaded available data in GenBank from China, Taiwan, Japan, Singapore, Italy and the USA [31]. The parsimony network was performed using TCS network inference method [47] in Population Analysis with Reticulate Trees (PopART) [48]. We also checked the number of haplotypes, $F_{S T}, N m$ and AMOVA using the same methodology described earlier.

In addition, a Bayesian clustering algorithm implemented in the program STRUCTURE v.2.3 was used to investigate genetic structure of individuals. The program was run under varying assumptions on Hardy-Weinberg (HW) and linkage equilibriums [49], with ten independent runs performed for each value of $K(K=1$ to 21$)$. In this analysis, the most likely number of genetic clusters
$(K)$ in the dataset is determined without prior information of the sampling locations, and then assigns proportion of the ancestry of each individual into the different clusters implemented in the program. The method of Evanno et al. [50] was used to determine the most likely number of clusters. This approach uses an ad hoc quantity, based on the second rate of change of the likelihood function between successive values of $K$. Poterior probability values were estimated using a Markov Chain Monte Carlo (MCMC) method and 1,000,000 interactions of each chain following the 100,000 iteration burn-in period were performed, as recommended by Pritchard et al. [49]. We visualized the partitioning of clusters using the program DISTRUCT [51].

\section{Results}

\section{Genetic diversity}

Partial sequences of the mtDNA cox1 (1337-bp) were amplified from 172 specimens, representing populations from Lao PDR $(n=155)$ and Thailand $(n=17)$. No insertions, deletions or stop codons were detected across all samples, which minimizes the likelihood of pseudogene amplification.

A total of 44 haplotypes were identified among the Lao populations (Table 1); of these, 13 haplotypes $(30 \%)$ were shared among Lao populations and 31 (70\%) were unique to single Lao populations. When the data was combined with Zhong et al. [31] (H1-H66) and the Thailand samples, a total of 46 haplotypes were found. These newly identified haplotypes are $\mathrm{H} 67-\mathrm{H} 112$ and were deposited in GenBank under accession numbers MN080720MN080765 (Table 1). Lao PDR sequences shared five haplotypes with Thailand, two haplotypes (H45 and H56) with the USA (California and Texas, respectively) and Thailand, and one haplotype (H46) with the USA (California) (Table 1).

Zhong et al. [31] amplified a fragment of 1433-bp of the mtDNA cox1, and identified 66 haplotypes of Ae. albopictus in 6 different countries (Italy, Japan, Taiwan, China, Singapore and the USA; 12 populations). The trimmed fragment we used is 96-bp smaller (1337-bp) than that of Zhong et al. [31]; however, no polymorphic sites were included in the trimmed sequence. Therefore, when we trimmed all fragments to 1377-bp, we still have the same 66 haplotypes as Zhong et al. [31] and 46 new haplotypes were recognized, totaling 112 haplotypes (Table 1).

The average number of nucleotide differences in $A e$. albopictus in Lao PDR populations ranged from 0.537 (LN) to $3.105(\mathrm{KM})$, corresponding with the range of the nucleotide diversity $(\pi) 0.00040(\mathrm{LN})$ to 0.00232 (KM). Haplotype diversity $(H d)$ ranged from $0.416 \pm 0.116$ (mean $\pm \mathrm{SD}$ ) (LN) to $0.942 \pm 0.029$ (VC) (Table 2). 
Table 1 Haplotypes of Aedes albopictus based on the mtDNA cox 1 marker

\begin{tabular}{|c|c|c|c|}
\hline Haplotype & $n$ & Country (code) & GenBank ID \\
\hline $\mathrm{HO}^{\mathrm{a}}$ & 6 & China (GZ, XM), USA (LA01) & KC690896 \\
\hline $\mathrm{HO}^{\mathrm{a}}$ & 5 & China (GZ) & KC690897 \\
\hline $\mathrm{HO}^{\mathrm{a}}$ & 113 & $\begin{array}{c}\text { China (GZ, XM, JS,), Taiwan (TW), Japan } \\
\text { (JP), Italy (IT), USA (LA01, LA11, HW) }\end{array}$ & KC690898 \\
\hline $\mathrm{HO}^{\mathrm{a}}$ & 3 & China (GZ) & KC690899 \\
\hline $\mathrm{HOS}^{\mathrm{a}}$ & 1 & China (GZ) & KC690900 \\
\hline $\mathrm{HO6}^{\mathrm{a}}$ & 2 & China (GZ) & KC690901 \\
\hline $\mathrm{H} 07^{\mathrm{a}}$ & 1 & China (XM) & KC690902 \\
\hline $\mathrm{HO}^{\mathrm{a}}$ & 9 & China (XM) & KC690903 \\
\hline $\mathrm{HO9}^{\mathrm{a}}$ & 1 & China (XM) & KC690904 \\
\hline $\mathrm{H} 10^{\mathrm{a}}$ & 1 & China (XM) & KC690905 \\
\hline $\mathrm{H} 11^{\mathrm{a}}$ & 1 & China (XM) & KC690906 \\
\hline $\mathrm{H} 12^{\mathrm{a}}$ & 2 & China (XM) & KC690907 \\
\hline $\mathrm{H} 13^{\mathrm{a}}$ & 2 & China (XM) & KC690908 \\
\hline $\mathrm{H} 14^{\mathrm{a}}$ & 1 & China (XM) & KC690909 \\
\hline $\mathrm{H} 15^{\mathrm{a}}$ & 1 & China (XM) & KC690910 \\
\hline $\mathrm{H} 16^{\mathrm{a}}$ & 7 & China (JS) & KC690911 \\
\hline $\mathrm{H} 17^{\mathrm{a}}$ & 26 & $\begin{array}{l}\text { Taiwan (TW), Italy (IT), USA (LA11, TX, } \\
\text { HW) }\end{array}$ & KC690912 \\
\hline $\mathrm{H} 18^{\mathrm{a}}$ & 1 & Taiwan (TW) & KC690913 \\
\hline $\mathrm{H} 19^{\mathrm{a}}$ & 2 & Taiwan (TW), USA (LA11) & KC690914 \\
\hline $\mathrm{H} 2 \mathrm{O}^{\mathrm{a}}$ & 1 & Taiwan (TW) & KC690915 \\
\hline $\mathrm{H} 21^{\mathrm{a}}$ & 1 & Taiwan (TW) & KC690916 \\
\hline $\mathrm{H} 22^{\mathrm{a}}$ & 3 & Taiwan (TW), USA (LA11) & KC690917 \\
\hline $\mathrm{H} 23^{\mathrm{a}}$ & 1 & Taiwan (TW) & KC690918 \\
\hline $\mathrm{H} 24^{\mathrm{a}}$ & 23 & Japan (JP), Singapore (SG) & KC690919 \\
\hline $\mathrm{H} 25^{\mathrm{a}}$ & 1 & Japan (JP) & KC690920 \\
\hline $\mathrm{H} 26^{\mathrm{a}}$ & 1 & Singapore (SG) & KC690921 \\
\hline $\mathrm{H} 27^{\mathrm{a}}$ & 8 & Singapore (SG) & KC690922 \\
\hline $\mathrm{H} 28^{\mathrm{a}}$ & 1 & Singapore (SG) & KC690923 \\
\hline $\mathrm{H} 29^{\mathrm{a}}$ & 1 & Singapore (SG) & KC690924 \\
\hline $\mathrm{H} 3 \mathrm{O}^{\mathrm{a}}$ & 2 & Singapore (SG) & KC690925 \\
\hline $\mathrm{H} 31^{\mathrm{a}}$ & 2 & Singapore (SG) & KC690926 \\
\hline $\mathrm{H} 32^{\mathrm{a}}$ & 1 & Singapore (SG) & KC690927 \\
\hline $\mathrm{H} 33^{\mathrm{a}}$ & 1 & Singapore (SG) & KC690928 \\
\hline $\mathrm{H} 34^{\mathrm{a}}$ & 1 & Singapore (SG) & KC690929 \\
\hline $\mathrm{H} 35^{\mathrm{a}}$ & 1 & Singapore (SG) & KC690930 \\
\hline $\mathrm{H} 36^{\mathrm{a}}$ & 1 & Italy (IT) & KC690931 \\
\hline $\mathrm{H} 37^{\mathrm{a}}$ & 40 & Italy (IT), USA (NJ, TX) & KC690932 \\
\hline $\mathrm{H} 38^{\mathrm{a}}$ & 1 & Italy (IT) & KC690933 \\
\hline $\mathrm{H} 39^{\mathrm{a}}$ & 6 & Italy (IT), USA (TX) & KC690934 \\
\hline $\mathrm{H} 40^{\mathrm{a}}$ & 2 & Italy (IT) & KC690935 \\
\hline $\mathrm{H} 41^{\mathrm{a}}$ & 4 & Italy (IT) & KC690936 \\
\hline $\mathrm{H} 42^{\mathrm{a}}$ & 1 & Italy (IT) & KC690937 \\
\hline $\mathrm{H} 43^{\mathrm{a}}$ & 1 & Italy (IT) & KC690938 \\
\hline $\mathrm{H} 44^{\mathrm{a}}$ & 1 & Italy (IT) & KC690939 \\
\hline $\mathrm{H} 45^{\mathrm{a}, \mathrm{b}}$ & 52 & $\begin{array}{l}\text { USA (LA01), Laos (BK, BO, CH, KM, LN, LP, } \\
\text { VC, XB), Thailand (TH) }\end{array}$ & KC690940 \\
\hline $\mathrm{H} 46^{\mathrm{a}, \mathrm{b}}$ & 5 & USA (LA01), Laos (BK, BO, CH) & KC690941 \\
\hline
\end{tabular}

Table 1 (continued)

\begin{tabular}{|c|c|c|c|}
\hline Haplotype & $n$ & Country (code) & GenBank ID \\
\hline $\mathrm{H} 47^{\mathrm{a}}$ & 1 & USA (LA01) & KC690942 \\
\hline $\mathrm{H} 48^{\mathrm{a}}$ & 2 & USA (LA01) & KC690943 \\
\hline $\mathrm{H} 49^{\mathrm{a}}$ & 7 & USA (LA11) & KC690944 \\
\hline $\mathrm{H} 50^{\mathrm{a}}$ & 1 & USA (LA11) & KC690945 \\
\hline $\mathrm{H} 51^{\mathrm{a}}$ & 2 & USA (NJ) & KC690946 \\
\hline $\mathrm{H} 52^{\mathrm{a}}$ & 4 & USA (NJ) & KC690947 \\
\hline $\mathrm{H} 53^{\mathrm{a}}$ & 2 & USA (NJ) & KC690948 \\
\hline $\mathrm{H} 54^{\mathrm{a}}$ & 2 & USA (NJ) & KC690949 \\
\hline $\mathrm{H} 55^{\mathrm{a}}$ & 3 & USA (TX) & KC690950 \\
\hline $\mathrm{H} 56^{\mathrm{a}, \mathrm{b}}$ & 5 & USA (TX), Laos (BK, BO), Thailand (TH) & KC690951 \\
\hline $\mathrm{H} 57^{\mathrm{a}}$ & 1 & USA (TX) & KC690952 \\
\hline $\mathrm{H} 58^{\mathrm{a}}$ & 1 & USA (TX) & KC690953 \\
\hline $\mathrm{H} 59^{\mathrm{a}}$ & 1 & USA (TX) & KC690954 \\
\hline $\mathrm{H} 6 \mathrm{O}^{\mathrm{a}}$ & 1 & USA (TX) & KC690955 \\
\hline $\mathrm{H} 61^{\mathrm{a}}$ & 16 & USA (HW) & KC690956 \\
\hline $\mathrm{H} 62^{\mathrm{a}}$ & 2 & USA (HW) & KC690957 \\
\hline$H 63^{\mathrm{a}}$ & 1 & USA (HW) & KC690958 \\
\hline $\mathrm{H} 64^{\mathrm{a}}$ & 1 & USA (HW) & KC690959 \\
\hline $\mathrm{H} 65^{\mathrm{a}}$ & 1 & USA (HW) & KC690960 \\
\hline$H 66^{\mathrm{a}}$ & 1 & USA (HW) & KC690961 \\
\hline $\mathrm{H} 67^{\mathrm{c}}$ & 1 & Lao PDR (BK) & MN080720 \\
\hline $\mathrm{H} 68^{\mathrm{c}}$ & 1 & Lao PDR (BK) & MN080721 \\
\hline $\mathrm{H} 69^{c}$ & 4 & Lao PDR (BK, CH), Thailand (TH) & MN080722 \\
\hline $\mathrm{H} 70^{c}$ & 1 & Lao PDR (BK) & MN080723 \\
\hline $\mathrm{H} 71^{\mathrm{C}}$ & 11 & Lao PDR (BK, BO, LP) & MN080724 \\
\hline $\mathrm{H} 72^{\mathrm{C}}$ & 1 & Lao PDR (BK) & MN080725 \\
\hline $\mathrm{H} 73^{\mathrm{C}}$ & 1 & Lao PDR (BK) & MN080726 \\
\hline $\mathrm{H} 74^{\mathrm{c}}$ & 1 & Lao PDR (BK) & MN080727 \\
\hline $\mathrm{H} 75^{\mathrm{C}}$ & 6 & Lao PDR (BO, CH, LN) & MN080728 \\
\hline$H 76^{c}$ & 20 & Lao PDR (BO, LN, XB), Thailand (TH) & MN080729 \\
\hline $\mathrm{H} 77^{\mathrm{c}}$ & 4 & Lao PDR (BO) & MN080730 \\
\hline $\mathrm{H} 78^{\mathrm{C}}$ & 2 & Lao PDR (CH) & MN080731 \\
\hline $\mathrm{H} 79^{\mathrm{c}}$ & 1 & Lao PDR (CH) & MN080732 \\
\hline $\mathrm{H} 80^{c}$ & 1 & Lao PDR (CH) & MN080733 \\
\hline $\mathrm{H} 81^{\mathrm{c}}$ & 1 & Lao PDR (CH) & MN080734 \\
\hline $\mathrm{H} 82^{c}$ & 1 & Lao PDR (CH) & MN080735 \\
\hline $\mathrm{H} 83^{\mathrm{C}}$ & 1 & Lao PDR (CH) & MN080736 \\
\hline $\mathrm{H} 84^{\mathrm{c}}$ & 3 & Lao PDR (CH, LP, VC) & MN080737 \\
\hline $\mathrm{H} 85^{\mathrm{c}}$ & 2 & Lao PDR (KM) & MN080738 \\
\hline $\mathrm{H} 86^{\mathrm{C}}$ & 6 & Lao PDR (KM, VC) & MN080739 \\
\hline $\mathrm{H} 87^{c}$ & 4 & Lao PDR (KM, VC) & MN080740 \\
\hline $\mathrm{H} 88^{\mathrm{C}}$ & 1 & Lao PDR (KM) & MN080741 \\
\hline $\mathrm{H} 89^{\mathrm{c}}$ & 2 & Lao PDR (KM) & MN080742 \\
\hline $\mathrm{H} 90^{c}$ & 3 & Lao PDR (KM, XB) & MN080743 \\
\hline $\mathrm{H} 91^{\mathrm{C}}$ & 1 & Lao PDR (LP) & MN080744 \\
\hline $\mathrm{H} 92^{\mathrm{C}}$ & 2 & Lao PDR (LP) & MN080745 \\
\hline $\mathrm{H} 93^{\mathrm{C}}$ & 2 & Lao PDR (LP, VC) & MN080746 \\
\hline $\mathrm{H} 94^{\mathrm{c}}$ & 1 & Lao PDR (LP) & MN080747 \\
\hline $\mathrm{H} 95^{\mathrm{c}}$ & 1 & Lao PDR (LP) & MN080748 \\
\hline
\end{tabular}


Table 1 (continued)

\begin{tabular}{|c|c|c|c|}
\hline Haplotype & $n$ & Country (code) & GenBank ID \\
\hline $\mathrm{H} 96^{\mathrm{C}}$ & 2 & Lao PDR (LP), Thailand (TH) & MN080749 \\
\hline $\mathrm{H} 97^{\mathrm{c}}$ & 1 & Lao PDR (LP) & MN080750 \\
\hline $\mathrm{H} 98^{\mathrm{c}}$ & 2 & Lao PDR (VC) & MN080751 \\
\hline $\mathrm{H} 99^{c}$ & 2 & Lao PDR (VC) & MN080752 \\
\hline $\mathrm{H} 100^{c}$ & 1 & Lao PDR (VC) & MN080753 \\
\hline $\mathrm{H} 101^{c}$ & 1 & Lao PDR (VC) & MN080754 \\
\hline $\mathrm{H} 102^{c}$ & 2 & Lao PDR (VC) & MN080755 \\
\hline $\mathrm{H} 103^{c}$ & 1 & Lao PDR (VC) & MN080756 \\
\hline $\mathrm{H} 104^{c}$ & 1 & Lao PDR (VC) & MN080757 \\
\hline $\mathrm{H} 105^{c}$ & 1 & Lao PDR (VC) & MN080758 \\
\hline $\mathrm{H} 106^{c}$ & 2 & Lao PDR (XB) & MN080759 \\
\hline $\mathrm{H} 107^{\mathrm{c}}$ & 4 & Lao PDR (XB) & MN080760 \\
\hline $\mathrm{H} 108^{c}$ & 1 & Thailand (TH) & MN080761 \\
\hline $\mathrm{H} 109^{c}$ & 2 & Thailand (TH) & MN080762 \\
\hline $\mathrm{H} 110^{c}$ & 3 & Thailand (TH) & MN080763 \\
\hline $\mathrm{H} 111^{\mathrm{c}}$ & 1 & Thailand (TH) & MN080764 \\
\hline $\mathrm{H} 112^{c}$ & 1 & Thailand $(\mathrm{TH})$ & MN080765 \\
\hline
\end{tabular}

a Haplotype data obtained from Zhong et al. [31]

${ }^{b}$ Shared haplotypes with Zhong et al. [31]

' New haplotypes

Abbreviations: GZ, Guangzhou; XM, Xiamen; JS, Jiangsu; TW, Xinzhu; JP, Nagazaki; SG, Helios Block; IT, Trentino; LA01, California; LA11, California; NJ, New Jersey; TX, Texas; HW, Hawaii; BK, Borikhamxay; BO, Bokeo; CH, Champasak; KM, Khammuane; LN, Luangnamtha; LP, Luang Prabang; VC, Vientiane Capital; XB, Xayabouly; $\mathrm{TH}$, Thailand

Table 2 Summary of haplotype and nucleotide diversity measures of the cox 1 gene for Ae. albopictus in Lao PDR

\begin{tabular}{llllll}
\hline $\begin{array}{l}\text { Population } \\
\text { code }\end{array}$ & $n$ & $\mathrm{H}$ & $\mathrm{Hd} \pm \mathrm{SD}$ & $\pi \pm \mathrm{SD}$ & $K$ \\
\hline BK & 20 & 11 & $0.874 \pm 0.064$ & $0.00150 \pm 0.0009$ & 2.000 \\
BO & 20 & 7 & $0.821 \pm 0.056$ & $0.00131 \pm 0.0009$ & 1.753 \\
CH & 20 & 11 & $0.805 \pm 0.090$ & $0.00110 \pm 0.0008$ & 1.468 \\
KM & 15 & 8 & $0.914 \pm 0.043$ & $0.00232 \pm 0.0010$ & 3.105 \\
LN & 20 & 3 & $0.416 \pm 0.116$ & $0.00040 \pm 0.0004$ & 0.537 \\
LP & 20 & 11 & $0.868 \pm 0.064$ & $0.00145 \pm 0.0009$ & 1.942 \\
VC & 20 & 12 & $0.942 \pm 0.029$ & $0.00220 \pm 0.0012$ & 2.937 \\
XB & 20 & 5 & $0.716 \pm 0.087$ & $0.00110 \pm 0.0007$ & 1.474 \\
\hline
\end{tabular}

Abbreviations: $\mathrm{n}$, number of individuals analyzed; $\mathrm{H}$, number of haplotypes; $\mathrm{Hd}$, haplotype diversity; SD, standard deviation; $\pi$, nucleotide diversity; $K$, average of nucleotide differences; BK, Borikhamxay; BO, Bokeo; $\mathrm{CH}$, Champasak; KM, Khammuane; LN, Luangnamtha; LP, Luang Prabang; VC, Vientiane Capital; XB, Xayabouly

The highest level of genetic differentiation in Lao PDR based on the fixation index $F_{S T}$ was between LN and LP $\left(F_{S T}=0.33288, P^{<} 0.05\right)$. Gene flow $(\mathrm{Nm})$ was $>1$ among all populations, except $\mathrm{LN}$ and $\mathrm{XB}$ (Table 3 ). When analyzed all together including the samples of Zhong et al. [31], the highest $F_{S T}$ was between LN (Luangnamtha, Lao
PDR) and JS (Jiangsu, China) $\left(F_{S T}=0.610, P^{<} 0.05\right)$ (Additional file 1: Table S1).

Global AMOVA tests indicated a high proportion of the total genetic variance was attributable to withinpopulation variation (85.98\%), suggesting low and significant genetic structure among populations $\left(F_{S T}=0.14\right.$, $P \leq 0.001)$ in Lao PDR. When we added all samples including that of Zhong et al. [31], global AMOVA found a significant overall population structure in Ae. albopictus $\left(F_{S T}=0.43, P \leq 0.001\right)$, with $56.8 \%$ of genetic variation found within-population and $43.2 \%$ among-populations. The spatial analysis of molecular variance (SAMOVA), based on mtDNA data, showed no genetically distinct population groups. Partitions of the sampling areas for each $K$ value were not informative. $F_{C T}$ values presented a narrow range between 0.18 and 0.23 . (Additional file 2: Figure S1). Mantel tests showed that genetic and geographical distances (Additional file 3: Table S2) among populations in Lao PDR do not support a pattern of isolation by distance $(r=0.0846, P=0.1433)$.

Assessment of population expansion based on neutrality test resulted primarily in negative values but most were not statistically significant, with the exception of Tajima's $D$ for $\mathrm{CH}$, and Fu's $F$ s for BK, $\mathrm{CH}, \mathrm{LN}$ and LP (Table 4). Mismatch distribution models revealed poor fit to equilibrium distribution (Additional file 4: Figure S2); both the sum of squared deviation (SSD) values $(0.016$, $P=0.29)$ and raggedness index (0.09) were not statistically significant in almost all the populations, except the SSD value for $\mathrm{BO}$ and $\mathrm{CH}$ and $\mathrm{Rag}$ for $\mathrm{CH}$ (Table 4), indicating further support for population expansion based on $\operatorname{cox} 1$ gene.

\section{Genetic relationships among haplotypes}

The parsimony network showed that the genealogical relationships among the haplotypes differed by 4-9 mutational steps (Fig. 2) and can be divided into three Groups: Group 1 mainly contained haplotypes from China, and a number of haplotypes from Japan, Italy, Taiwan and the USA; Group 2 contained haplotypes from China, Japan, Italy, Taiwan, the USA, and $50 \%$ of the haplotypes in Singapore; and Group 3 contained haplotypes from Lao PDR, Thailand, the remaining 50\% from Singapore, and three haplotypes shared with the USA. The most common haplotypes were $3(n=113)$ and $45(n=52)$ (Fig. 2, Table 2). Haplotype 3 was shared among populations from China, Taiwan, Japan, Italy and the USA, while H45 was shared among the USA, Thailand and all populations from Lao PDR (Fig. 2, Table 1).

\section{Genetic clustering of individuals}

Bayesian inference implemented in STRUCTURE revealed that the optimal partitioning of all Ae. albopictus 
Table 3 Pairwise differentiation ( $F_{S T}$, below the diagonal), and gene flow ( $N_{m}$, above the diagonal) among populations of Ae. albopictus in Lao PDR

\begin{tabular}{lllllllll}
\hline & BK & BO & CH & KM & LN & LP & VC \\
\hline BK & - & 6.50505 & $\infty$ & 12.2500 & 3.47518 & 5.69707 & 6.08466 \\
BO & $0.07138^{*}$ & - & 4.42693 & 4.62039 & 1.51515 & 3.83055 & 4.06553 & 2.33385 \\
CH & -0.00838 & $0.10148^{* *}$ & - & 8.32182 & 7.27273 & 3.02857 & 4.20786 & 1.50360 \\
KM & 0.04122 & $0.09765^{* *}$ & $0.05668^{*}$ & - & 1.65733 & 4.48241 & 25.51247 & 2.50000 \\
LN & $0.12578^{* *}$ & $0.24812^{* *}$ & 0.06433 & $0.23177^{* *}$ & - & 1.00205 & 1.19722 & 0.84646 \\
LP & $0.08068^{*}$ & $0.11546^{* *}$ & $0.14170^{* *}$ & $0.10035^{* *}$ & $0.33288^{* *}$ & - & 5.04399 & 1.95201 \\
VC & $0.07593^{* *}$ & $0.10952^{* *}$ & $0.10621^{* *}$ & 0.01922 & $0.29460^{* *}$ & $0.09019^{* *}$ & - & 2.49604 \\
XB & $0.17644^{* *}$ & 0.04544 & $0.20364^{* *}$ & $0.16667^{* *}$ & $0.37135^{* *}$ & $0.20391^{* *}$ & $0.16689^{*}$ & - \\
\hline
\end{tabular}

* Significant values after Bonferroni correction (* $\left.P^{<} 0.05, * * P^{<} 0.01\right)$

Abbreviations: BK, Borikhamxay; BO, Bokeo; CH, Champasak; KM, Khammuane; LN, Luangnamtha; LP, Luang Prabang; VC, Vientiane Capital; XB, Xayabouly

Table 4 Neutrality test and mismatch distribution of cox 1 gene of Ae. albopictus in Lao PDR

\begin{tabular}{llllll}
\hline Population code & \multicolumn{2}{c}{ Neutrality tests } & & \multicolumn{2}{c}{ Mismatch analysis } \\
\cline { 2 - 3 } \cline { 6 - 7 } & $D$ & $F_{S}$ & & SSD & Rag \\
\hline BK & -1.25890 & $-6.08335^{*}$ & & 0.012 & 0.056 \\
BO & 0.11573 & -1.61256 & & $0.040^{*}$ & 0.144 \\
CH & $-2.03130^{*}$ & $-8.03746^{*}$ & & $0.017^{*}$ & $0.131^{*}$ \\
KM & -1.10557 & -1.58578 & & 0.017 & 0.059 \\
LN & -0.97524 & $-0.07875^{*}$ & & 0.000 & 0.132 \\
LP & 0.47276 & $-6.25889^{*}$ & & 0.008 & 0.046 \\
VC & 0.54226 & -5.33883 & 0.007 & 0.038 \\
XB & 0.88892 & -0.12444 & 0.028 & 0.144 \\
Mean & -0.41892 & -3.64001 & 0.016 & 0.09 \\
\hline
\end{tabular}

* Significant value, $P^{<} 0.01$

Abbreviations: BK, Borikhamxay; BO, Bokeo; $\mathrm{CH}$, Champasak; KM, Khammuane; LN, Luangnamtha; LP, Luang Prabang; VC, Vientiane Capital; XB, Xayabouly

samples (China, Taiwan, Japan, Singapore, Italy, USA from Zhong et al. [31], Lao PDR and Thailand) was $K=8$. The individuals analyzed from the 21 populations were assigned to eight clusters with a certain probability value (Fig. 3, Additional file 5: Table S3). Most individuals from Lao PDR and Thailand were represented in clusters 1 and 2, and partially in clusters 4 and 8, sharing with Singapore, Japan and the USA (California samples). Samples from China were mainly found in clusters 3, 6 and 7, sharing with USA and Italy, and cluster 5 included the highest proportion of individuals from the USA (New Jersey and Texas samples: 86 and $72 \%$, respectively), as observed in Zhong et al. [31].

\section{Discussion}

The barcoding mitochondrial gene, cox 1 , has been widely used to analyze genetic diversity in Ae. albopictus [31, 52-59]. In this study, we followed the recommendation of Goubert et al. [60] that reviewed the literature on the use of the $\operatorname{cox} 1$ for population genetic studies, and employed a longer mtDNA marker designed by Zhong et al. [31].

\section{Genetic diversity in Ae. albopictus from Lao PDR}

Overall, we detected very high haplotype diversity in Ae. albopictus in Lao PDR, with 44 haplotypes identified from only eight populations. Among them, 13 haplotypes were shared (Table 1), in some cases by all eight populations. Low and significant genetic structure (Table 3) were observed, supporting the finding of other studies $[55,57,59,61]$.

The higher and significant differentiation among LN (Luangnamtha) and other locations in Lao PDR $\left(F_{S T}\right.$ 0.126-0.371), except $\mathrm{CH}$ (Champasak), may be due to climate (Table 3). Indeed, while Lao PDR has a predominantly tropical climate, the mountainous topography and the extensive Mekong River network in the northern and southern regions, results in variation in average temperature conditions and creates significantly different microclimates that may be highly relevant to mosquito development. On the other hand, the Mantel test revealed no correlation between genetic and geographical distances, indicating no isolation by distance of $A e$. albopictus in Lao PDR. Similar results were observed within countries [60, 62-65], except in Schmidt et al. [66]; they analyzed genetic structure of Ae. albopictus from 12 localities in China using single nucleotide polymorphism (SNPs) and found evidence for IBD.

Signs of recent expansion observed in Ae. albopictus across Lao PDR are evidenced by economic development, which is characterized by high rates of urbanization in the Association of South East Asian Nations (ASEAN) community. This has led to a better road infrastructure throughout the country and has increased connectivity between all the provinces, which has the potential to facilitate human-assisted movement of Aedes mosquitoes (SM, personal observation) and their pathogens 


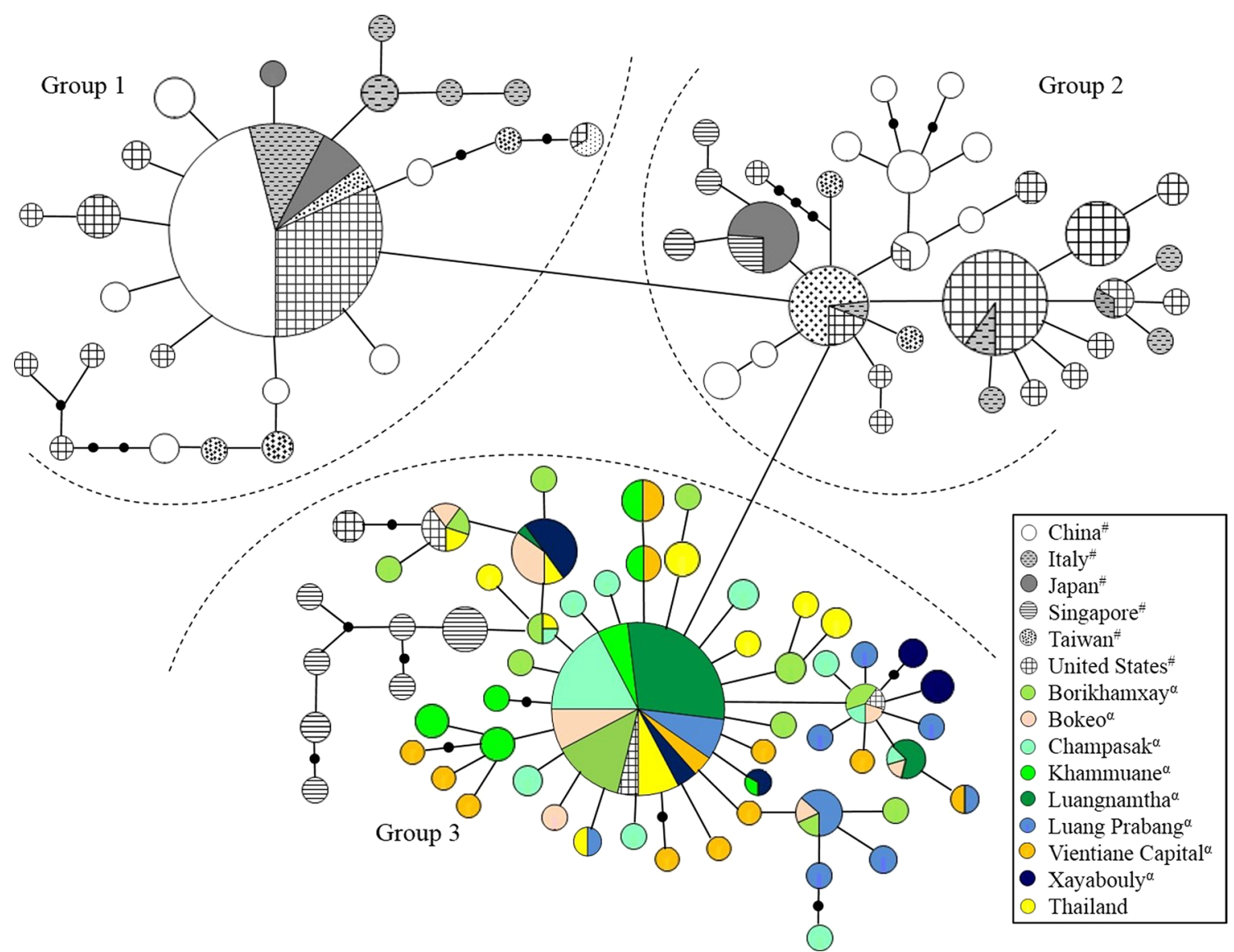

Fig. 2 Phylogenetic network of 112 mitochondrial haplotypes (1337 bp) of the cox 1 gene in Ae. albopictus. Localities are indicated by different colors (bottom-right). The area of each circle is approximately proportional to the frequency of the haplotype. \#Samples available in Genbank from Zhong et al. [31]. ' Samples from Lao PDR

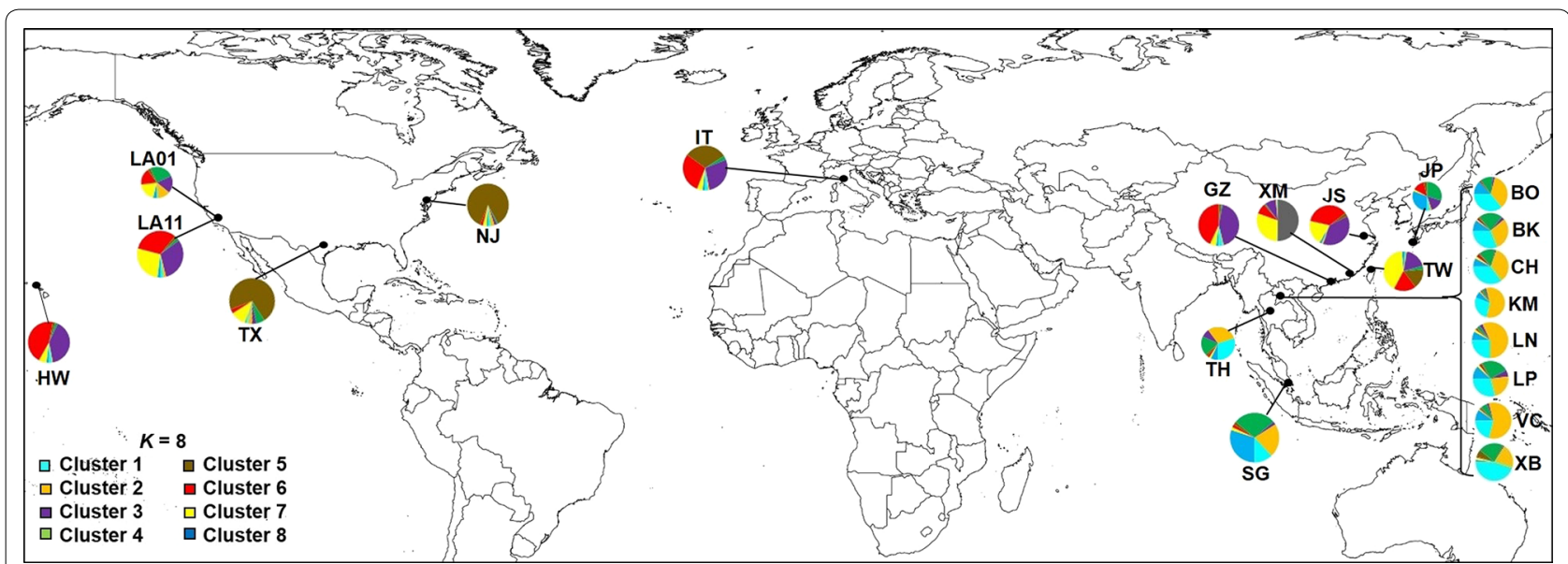

Fig. 3 Pie charts representing the proportional membership of Ae. albopictus identified in Bayesian cluster analysis (optimal partitioning of all samples, K=8). Abbreviations: China: GZ, Guangzhou; XM, Xiamen; JS, Jiangsu; Taiwan: TW, Xinzhu; Japan: JP, Nagazaki; Singapore: SG, Helios Block; Italy: IT, Trentino; USA: LA01, California; LA11, California; NJ, New Jersey; TX, Texas; and HW, Hawaii - are samples from Zhong et al. [31]; Lao PDR: BK, Borikhamxay; BO, Bokeo; CH, Champasak; KM, Khammuane; LN, Luangnamtha; LP, Luang Prabang; VC, Vientiane Capital; XB, Xayabouly; Thailand: TH, Thailand 
$[13,67]$. In addition, rubber plantations provided several potential breeding sites for Ae. albopictus including latexcollection cups $[68,69]$. According to Tangena et al. [12], the risk of dengue infection in natural forests and rubber plantations is higher than in northern region villages in Luang Prabang Province. Aedes albopictus is highly adaptable and successfully spread from its preferred forested environments to different rural and urban habitats, which has increased its potential as a vector and, consequently, arboviruses transmission risk in these more populated areas.

\section{Genetic relationship among Ae. albopictus in Lao PDR and the available haplotypes from other geographical regions and genetic clustering of individuals}

When populations of Ae. albopictus were analyzed from many different geographical regions, three haplotypes were observed to be shared between Lao PDR and other countries. H45 and H46 were shared with the USA (California) and Thailand, and H56 with the USA (Texas). $\mathrm{H} 45$ and $\mathrm{H} 46$ are shared haplotypes from Los Angeles, California, where samples were collected in 2001. Similarly, Zhong et al. [31] observed those haplotypes were shared with Singaporean populations and were not found in their collection in 2011; hence, the authors suggested that only specimens from subtropical/temperate climates could have established successfully in the USA. In addition, the $F_{S T}$ was lower when comparing Lao PDR with the 2001 California samples $(0.093-0.323, P \leq 0.05)$ than the ones collected 10 years later in $2011(0.286-0.529, P$ $\leq 0.05$ ) (Additional file 1: Table S1).

The phylogenetic network and the Bayesian cluster analyses corroborated the results from Zhong et al. [31]. Groups 1 and 2 (in the network analysis) and clusters 3, 5-7 (in the Bayesian analysis) included samples from temperate regions and most of group 3 and clusters 1, 2, 4, 8 (network and Bayesian analyses, respectively) included the majority of samples from tropical/ subtropical regions (Figs. 2, 3). Allozyme studies have shown that populations of Ae. albopictus from Japan are likely distinct from the remaining samples in the world [70] and Southeast Asia (Borneo, peninsula Malaysia) and southern Asian populations (India, Sri Lanka) can both be differentiated from northern Asian populations (China, Japan) [71]. Worldwide mitogenome diversity of Ae. albopictus was studied and three major haplogroups were found; the first haplogroup was mostly distributed in tropical regions, the second in temperate regions and the third appeared to be important in the spread of $A e$. albopictus from Asia [61]. A possible explanation for these differences is the presence of a photoperiodic diapause in Ae. albopictus from temperate regions [72-74], and absence of diapause among Ae. albopictus in tropical regions, such as in Brazil [72]. However, it is worth noting that the Singapore population represents a particular case in Southeast Asia. Its population is genetically connected both with tropical and temperate strains (Figs. 2, $3)$.

Although no study has performed a comprehensive analysis of the species' full native range [60], the genetic differentiation of native Asian populations of Ae. albopictus may confer both north-south (Korea to Indonesia) and east-west (Japan to India) pattern of genetic differentiation [61]; our results partially support the pattern of north-south as in Battaglia et al. [61].

Overall, we observed significant population structure in Ae. albopictus $\left(F_{S T}=0.43, P \leq 0.001\right)$. Similar results were observed in Zhong et al. [31] and Maynard et al. [75]. As mentioned, Zhong et al. [31] analyzed $\operatorname{cox} 1$ of Ae. albopictus from China, Taiwan, Japan, Singapore, Italy and the USA. Maynard et al. [75] using both microsatellite and mitochondrial markers observed significant relationship between genetic variability and geographical distance, but weak correlation in Ae. albopictus of IndoPacific regions.

Laotian Ae. albopictus populations were found to be very genetically related to the tropical Thailand strain. An allozyme study suggested that populations of Ae. albopictus in the eastern USA possibly originated from temperate Asian regions [67], while mtDNA variations revealed that populations in Represa do Congo and Sao Luis in Brazil formed a lineage paraphyletic to tropical Southeast Asian lineages, such as Cambodia, Vietnam, Thailand $[52,76]$ and likely Lao PDR.

\section{Conclusions}

To our knowledge, this study represents the first genetic analysis of Ae. albopictus in Lao PDR. Laotian Ae. albopictus are genetically related to populations from tropical/ subtropical regions. The high polymorphism but shallow population structure across Lao PDR and signs of a recent population expansion in Ae. albopictus may be the result of recent economic development that facilitates human-mediated movement of Ae. albopictus. We suggest that extensive movement and likely common reintroductions of Ae. albopictus to treated sites represent a major challenge to dengue control in Lao PDR.

\section{Supplementary information}

Supplementary information accompanies this paper at https://doi. org/10.1186/s13071-019-3740-0.

Additional file 1: Table S1. Pairwise differentiation, $F_{S T}$, among populations of Ae. albopictus. Abbreviations: China: GZ, Guangzhou; XM, Xiamen; JS, Jiangsu; Taiwan: TW, Xinzhu; Japan: JP, Nagazaki; Singapore: SG, Helios Block; Italy: IT, Trentino; USA: LA01, California; LA11, California; NJ, New 
Jersey; TX, Texas; HW, Hawaii; Lao PDR: BK, Borikhamxay; BO, Bokeo; CH, Champasak; KM, Khammuane; LN, Luangnamtha; LP, Luang Prabang; VC, Vientiane Capital; XB, Xayabouly; Thailand: TH, Thailand. "Samples available in GenBank from Zhong et al. [31]. *Significant values after Bonferroni correction $\left(P^{<} 0.05\right)$.

Additional file 2: Figure S1. Fixation indices obtained by SAMOVA for the best-clustering option at each pre-defined values of $K$. Abbreviations: $F_{C T}$. variation among groups of populations; $F_{S c}$ variation among populations within groups; $F_{S T}$, variation among population among groups.

Additional file 3: Table S2. Geographical distances (in km) among Ae. albopictus from Lao PDR. Abbreviations: BK, Borikhamxay; BO, Bokeo; CH, Champasak; KM, Khammuane; LN, Luangnamtha; LP, Luang Prabang; VC, Vientiane Capital; XB, Xayabouly.

Additional file 4: Figure S2. Mismatch distributions showing the frequencies of pairwise differences of Ae. albopictus in Lao PDR.

Additional file 5: Table S3. Assignment of the Bayesian clustering analysis of Ae. albopictus populations. Abbreviations: China: GZ, Guangzhou; XM, Xiamen; JS, Jiangsu; Taiwan: TW, Xinzhu; Japan: JP, Nagazaki; Singapore: SG, Helios Block; Italy: IT, Trentino; USA: LA01, California; LA11, California; NJ, New Jersey; TX, Texas; HW, Hawaii-are samples from Zhong et al. [31]; Lao PDR: BK, Borikhamxay; BO, Bokeo; CH, Champasak; KM, Khammuane: LN, Luangnamtha; LP, Luang Prabang; VC, Vientiane Capital; XB, Xayabouly; Thailand: TH, Thailand. "Samples available in Genbank from Zhong et al. [31]. The coefficient values above 0.25 are highlighted in bold.

\section{Acknowledgements}

Our sincere thanks to the public health officers from the eight provinces for helping us in the fieldwork, and to the Ministry of Public Health of Lao PDR for facilitating this research. We are grateful to Ms. Somphat Nilaxay and Mr. Santi Vilayheuang for assistance and Dr Moritoshi Iwagami and Mr. Lavy Lorphachan for helping with sequencing. We appreciate the reviewers for providing helpful comments and suggestions.

\section{Authors' contributions}

MTM conceived the study with input from PTB and SM. MTM performed the experiments and analysed the data with input from DMF and BDS. MTM EFM, PT, SC, NP, PK and SM contributed to the field collection or acquisition of samples. MTM drafted the manuscript. DMF, BDS, JCH, PTB and SM revised the manuscript. All authors read and approved the final manuscript.

\section{Funding}

The views expressed in this publication are those of the author and do not necessarily reflect the official policy or position of the Department of the Navy, Department of Defense, nor the U.S. Government. This study was partially supported by the U.S. Naval Medical Research Unit TWO, work unit number D1428, in support of the Military Infectious Diseases Research Program and Institut Pasteur du Laos. I (JCH) am a military service member. This work was prepared as part of my official duties. Title 17, U.S.C., \$105 provides that copyright protection under this title is not available for any work of the U.S. Government. Title 17, U.S.C., \$101 defines a U.S. Government work as a work prepared by a military service member or employee of the U.S. Government as part of that person's official duties.

\section{Availability of data and materials}

All data generated or analysed during the present study are included in this published article or available from the GenBank under the accession numbers MN080720-MN080765.

\section{Ethics approval and consent to participate}

Not applicable.

\section{Consent for publication}

Not applicable.

\section{Competing interests}

The authors declare that they have no competing interests.

\section{Author details}

${ }^{1}$ Medical Entomology and Vector Borne Disease Unit, Institut Pasteur du Laos, Vientiane, Lao People's Democratic Republic. ${ }^{2}$ Vysnova Partners Inc., Landover, MD, USA. ${ }^{3}$ Walter Reed Biosystematics Unit, Smithsonian Institution, Museum Support Center, MRC-534, Suitland, MD, 20746, USA. ${ }^{4}$ Center for Vector Biology and Department of Entomology, Rutgers University, New Brunswick, NJ, USA. ${ }^{5}$ Université Pierre et Marie Curie, Cellule Pasteur UPMC, Paris, France. ${ }^{6}$ Insect-Virus Interactions Group, Department of Genomes and Genetics, Institut Pasteur, Paris, France. ${ }^{7}$ Centre National de la Recherche Scientifique, Unité Mixte de Recherche 2000, Paris, France. ${ }^{8}$ Department of Epidemiology, Faculty of Public Health, University of Sao Paulo, Sao Paulo, Brazil. ${ }^{9}$ U.S. Naval Medical Research Unit TWO, Singapore, Singapore. ${ }^{10}$ Mahidol University, Bangkok, Thailand.

Received: 21 June 2019 Accepted: 4 October 2019

Published online: 14 October 2019

\section{References}

1. Undurraga EA, Halasa YA, Shepard DS. Use of expansion factors to estimate the burden of dengue in Southeast Asia: a systematic analysis. PLoS Negl Trop Dis. 2013;7:e2056.

2. Banu S, Hu W, Guo Y, Naish S, Tong S. Dynamic spatiotemporal trends of dengue transmission in the Asia-Pacific region, 1955-2004. PLoS ONE. 2014;9:e89440.

3. Khampapongpane B, Lewis HC, Ketmayoon P, Phonekeo D, Somoulay $\checkmark$, Khamsing A, et al. National dengue surveillance in the Lao People's Democratic Republic, 2006-2012: epidemiological and laboratory findings. Western Pac Surveill Response J. 2014;5:7-13.

4. Bounlu K, Tadano M, Makino Y, Arakaki K, Fukunaga T. A seroepidemiological study of dengue and Japanese encephalitis virus infections in Vientiane, Lao PDR. Jpn J Trop Med Hyg. 1992;38:172-80.

5. Fukunaga T, Phommasack B, Bounlu K, Saito M, Tadano M, Makino Y, et al. Epidemiological situation of dengue infection in Lao PDR. Trop Med. 1994;35:219-27.

6. Makino Y, Saito M, Phommasack B, Vongxay P, Kanemura K, Pothawan T, et al. Arbovirus infections in pilot areas in Laos. Trop Med. 1995;36:131-9.

7. Guo X, Zhao Q, Wu C, Zuo S, Zhang X, Jia N, Liu J, Zhou H, Zhang J. First isolation of dengue virus from Lao PDR in a Chinese traveler. Virol J. 2013;10:70.

8. Lao M, Caro V, Thiberge JM, Bounmany P, Vongpayloth K, et al. Co-circulation of dengue virus type 3 genotypes in Vientiane Capital, Lao PDR. PLoS ONE. 2014;9:e115569.

9. WHO. Dengue guideline for diagnosis, treatment, prevention and control. Geneva: World Health Organization; 2009. http://www.who.int/tdr/publi cations/training-guideline-publications/dengue-diagnosis-treatment/ en/. Accessed 10 Jan 2019.

10. WHO. Update on dengue situation in the Western Pacific Region. Geneva: World Health Organization; 2017. http://www.wpro.who.int/emerg ing_diseases/dengue_biweekly_report_20171024.pdf. Accessed 10 Jan 2019.

11. Tangena JAA, Thammavong $P$, Malaithong N, Inthavong T, Ouanesamon P, Brey PT, Lindsay SW. Diversity of mosquitoes (Diptera: Culicidae) attracted to human subjects in rubber plantations, secondary forests, and villages in Luang Prabang province, Northern Lao PDR. J Med Entomol. 2017:54:1589-604.

12. Tangena JAA, Thammavong P, Lindsay SW, Brey PT. Risk of exposure to potential vector mosquitoes for rural workers in northern Lao PDR. PLoS Negl Trop Dis. 2017;211:e0005802

13. Gratz NG. Critical review of the vector status of Aedes albopictus. Med Vet Entomol. 2004:18:215-27.

14. Benedict MQ, Levine RS, Hawley WA, Lounibos LP. Spread of the tiger: global risk of invasion by the mosquito Aedes albopictus. Vector Borne Zoonotic Dis. 2007;7:76-85

15. Lambretchts L, Scott TW, Gubler DJ. Consequences of the expanding global distribution of Aedes albopictus for dengue virus transmission. PLoS Negl Trop Dis. 2010:4:e646.

16. Kraemer MU, Sinka ME, Duda KA, Mylne AQ, Shearer FM, Barker CM, et al. The global distribution of the arbovirus vectors Aedes aegypti and $A e$. albopictus. eLife. 2015;4:e08347. 
17. Reiter P, Sprenger D. The used tire trade: a mechanism for the worldwide dispersal of container breeding mosquitoes. J Am Mosq Control Assoc. 1987;3:494-501.

18. Coffey LL, Failloux AB, Weaver SC. Chikungunya virus-vector interactions. Viruses. 2014;6:4628-63.

19. Paupy C, Ollomo B, Kamgang B, Mountailler S, Rousset D, Demanou $\mathrm{M}$, et al. Comparative role of Aedes albopictus and Aedes aegypti in the emergence of dengue and chikungunya in Central Africa. Vector Borne Zoonotic Dis. 2010;10:259-66.

20. Tomasello D, Schlagenhauf P. Chikungunya and dengue autochthonous cases in Europe, 2007-2012. Travel Med Infect Dis. 2013;11:274-84.

21. Lai S, Huang Z, Zhou H, Anders KL, Perkins TA, Yin W, et al. The changing epidemiology of dengue in China, 1990-2014: a descriptive analysis of 25 years of nationwide surveillance data. BMC Med. 2015;13:100.

22. Rosen L, Roseboom LE, Gubler DJ, Lien JC, Chaniotis BN. Comparative susceptibility of mosquito species and strains to oral and parenteral infection with dengue and Japanese encephalitis viruses. Am J Trop Med Hyg. 1985;34:603-15.

23. Vega-Rua A, Zouache K, Caro V, Diancourt L, Delaunay P, Grandadam $M$, et al. High efficiency of temperate Aedes albopictus to transmit chikungunya and dengue viruses in the southeast of France. PLOS ONE. 2013:8:e59716.

24. Mendenhall IH, Manuel M, Moorthy M, Lee TTM, Low DHW, Misse D, et al. Peridomestic Aedes malayensis and Aedes albopictus are capable vectors of arboviruses in cities. PLoS Negl Trop Dis. 2017;11:e0005667.

25. Wong PSJ, Li MZI, Chong CS, Ng LC, Tan CH. Aedes (Stegomyia) albopictus (Skuse): a potential vector of Zika virus in Singapore. PLoS Negl Trop Dis. 2013;7:e2348

26. Grard G, Caron M, Mombo IM, Nkoghe D, Mboui Ondo S, Jiolle D, et al. Zika virus in Gabon (Central Africa) - 2007: a new threat from Aedes albopictus? PLoS Negl Trop Dis. 2014;8:e2681.

27. Gardner LM, Chen N, Sarkar S. Global risk of Zika virus depends critically on vector status of Aedes albopictus. Lancet Infect Dis. 2016;16:522-3.

28. Moreira LA, Iturbe-Ormaetxe I, Jeffery JA, Lu G, Pyke AT, Hedges LM, et al. A Wolbachia symbiont in Aedes aegypti limits infection with dengue, chikungunya, and Plasmodium. Cell. 2009;139:1268-78.

29. Campos M, Spenassatto C, Da Graca Macoris ML, Paduan KS, Pinto J, Ribolla PE. Seasonal population dynamics and the genetic structure of the mosquito vector Aedes aegypti in Sao Paulo, Brazil. Ecol Evol. 2012;2:2794-802.

30. Rasic G, Filipovic I, Weeks AR, Hoffmann AA. Genome-wide SNPs lead to strong signals of geographic structure and relatedness patterns in the major arbovirus vector, Aedes aegypti. BMC Genomics. 2014;15:275.

31. Zhong D, Lo E, Metzger ME, Cummings R, Bonizzoni M, Fujioka KK, et al. Genetic analysis of invasive Aedes albopictus populations in Los Angeles County, California and its potential public health impact. PLOS ONE. 2013;8:e68586.

32. Rattanarithikul R, Harbach RE, Harrison BA, Panthusiri P, Coleman RE, Richardson JH. Ilustrated keys to the mosquitoes of Thailand VI. Tribe Aedini. Southeast Asian J Trop Med. 2010;41(Suppl. 1):1-225.

33. Kearse M, Moir R, Wilson A, Stones-Havas S, Cheung M, Sturrock S, et al. Geneious basic: an integrated and extendable desktop software platform for the organization and analysis of sequence data. Bioinformatics. 2012;28:1647-9.

34. Librado P, Rozas J. DnaSP v5: a software for comprehensive analysis of DNA polymorphism data. Bioinformatics. 2009;25:1451-2.

35. Nei M. Analysis of gene diversity in subdivided populations. Proc Natl Acad Sci USA. 1973;70:3321-3.

36. Excoffier L, Laval G, Schneider S. Arlequin ver. 3.0: an integrated software package for population genetics data analysis. Evol Bioinform Online. 2005;1:47-50

37. Dupanloup I, Schneider S, Excoffier L. A simulated annealing approach to define the genetic structure of populations. Mol Ecol. 2002;11:2571-81.

38. Mantel N. The detection of disease clustering and a generalized regression approach. Cancer Res. 1967;27:209-20.

39. Peakall R, Smouse PE. GenAlEx 6: genetic analysis in Excel. Population genetic software for teaching and research. Mol Ecol Notes. 2006:6:288-95.

40. Peakall R, Smouse PE. GenAlEx 6.5: genetic analysis in Excel. Population genetic software for teaching and research-an update. Bioinformatics. 2012:28:2537-9.
41. Tajima F. Statistical method for testing the neutral mutation hypothesis by DNA polymorphism. Genetics. 1989;123:585-95.

42. Fu YX. Statistical tests of neutrality of mutations against population growth, hitchhiking and background selection. Genetics. 1997;147:915-25.

43. Slatkin M, Hudson RR. Pairwise comparisons of mitochondrial DNA sequences in stable and exponentially growing populations. Genetics. 1991;129:555-62

44. Rogers AR, Harpending H. Population growth makes waves in the distribution of pairwise differences. Mol Biol Evol. 1992;9:552-69.

45. Rogers AR. Genetic evidence for a Pleistocene population explosion. Evolution. 1995:49:608-15.

46. Zarza E, Reynoso VH, Emerson BC. Diversification in the northern neotropics: mitochondrial and nuclear DNA phylogeography of the iguana Ctenosaura pectinata and related species. Mol Ecol. 2008;17:3259-75.

47. Clement M, Pousada D, Crandall KA. TCS: a computer program to estimate gene genealogies. Mol Ecol. 2000;9:1657-9.

48. Leigh JW, Bryant D. PopART: full-feature software for haplotype network construction. Methods Ecol Evol. 2015;6:1110-6.

49. Pritchard JK, Stephens M, Donnelly P. Inference of population structure using multilocus genotype data. Genetics. 2000;155:945-59.

50. Evanno G, Regnaut S, Goudet J. Detecting the number of clusters of individuals using the software STRUCTURE: a simulation study. Mol Ecol. 2005;14:2611-20.

51. Rosenberg NA. Disrupt: a program for the graphical display of population structure. Mol Ecol Notes. 2004;4:137-8.

52. Mousson L, Dauga C, Garrigues T, Schaffner F, Vazeille M, Failloux AB. Phylogeography of Aedes (Stegomyia) aegypti (L.) and Aedes (Stegomyia) albopictus (Skuse) (Diptera: Culicidae) based on mitochondrial DNA variations. Genet Res. 2005;86:1-11.

53. Delatte H, Bagny L, Brengue C, Bouetard A, Paupy C, Fontenille D. The invaders: phylogeography of dengue and chikungunya viruses Aedes vectors, on the South West islands of the Indian Ocean. Infect Genet Evol. 2011;11:1769-81.

54. Zitko T, Kovacic A, Desdevises Y, Puizina J. Genetic variation in East-Adriatic populations of the Asian tiger mosquito, Aedes albopictus (Diptera: Culicidae), inferred from NADH5 and COI sequence variability. Eur J Entomol. 2011;108:501-8.

55. Porretta D, Mastrantonio V, Bellini R, Somboon P, Urabanelli S. Glacial history of a modern invader: phylogeography and species distribution modelling of the Asian tiger mosquito Aedes albopictus. PLOS ONE. 2013:7:e44515

56. Beebe NW, Ambrose L, Hill LA, Davis JB, Hapgood G, Cooper RD, et al. Tracing the tiger: population genetics provides valuable insights into the Aedes (Stegomyia) albopictus invasion of the Australasian region. PLoS Negl Trop Dis. 2013;7:e2361.

57. Zawani MKN, Abu HA, Sazaly AB, Zary SY, Darlina MN. Population genetic structure of Aedes albopictus in Penang, Malaysia. Gen Mol Res. 2014;13:8184-96.

58. Futami K, Valderrama A, Baldi M, Minakawa N, Marin Rodrigues R, Chaves LF. New and common haplotypes shape genetic diversity in Asian tiger mosquito populations from Costa Rica and Panama. J Econ Entomol. 2015:108:761-8.

59. Ismail N-A, Dom NC, Ismail R, Ahmad AH, Zaki A, Camalxaman SN. Mitochondrial cytochrome oxidase I gene sequence analysis of Aedes albopictus in Malaysia. J Am Mosq Control Assoc. 2015;31:305-12.

60. Goubert C, Minard G, Vieira C, Boulesteix M. Population genetics of the Asian tiger mosquito Aedes albopictus, an invasive vector of human diseases. Heredity. 2016;117:125-34.

61. Battaglia V, Gabrieli P, Brandini S, Capodiferro MR, Javier PA, Chen XG, et al. The worldwide spread of the tiger mosquito as revealed by mitogenome haplogroup diversity. Front Genet. 2016;7:208.

62. Wellmer H. Dengue haemorrhagic fever in Thailand. Geomedical observations on developments over the period 1970-1979. Berlin: Springer; 1983.

63. Paupy C, Girod R, Salvan M, Rodhain F, Failloux AB. Population structure of Aedes albopictus from La Reunion Island (Indian Ocean) with respect to susceptibility to a dengue virus. Heredity. 2001;87:273-83.

64. Vazeille M, Mousson L, Rakatoarivony I, Villeret R, Rodhain F, Duchemin $\mathrm{J}$-B, et al. Population genetic structure and competence as a vector for 
dengue type 2 virus of Aedes aegypti and Aedes albopictus from Madagascar. Am J Trop Med Hyg. 2001;65:491-7.

65. Maia R, Scarpassa V, Maciel-Litaiff L, Tadei W. Reduced levels of genetic variation in Aedes albopictus (Diptera: Culicidae) from Manaus, Amazonas State, Brazil, based on analysis of the mitochondrial DNA ND5 gene. Gen Mol Res. 2009;8:998-1007.

66. Schmidt TL, Rasic G, Zhang D, Zheng X, Xi Z, Hoffmann AA. Genome-wide SNPs reveal the drivers of gene flow in an urban population of the Asian tiger mosquito, Aedes albopictus. PLoS Negl Trop Dis. 2017;1 1:e0006009.

67. Novak R. The Asian tiger mosquito, Aedes albopictus. Wing Beats. 1992;3:5.

68. Paily KP, Chandhiran K, Vanamail P, Kumar NP, Jambulingam P. Efficacy of a mermithid nematode Romanomermis iyengari (Welch) (Nematoda: Mermithidae) in controlling tree hole-breeding mosquito Aedes albopictus (Skuse) (Diptera: Culicidae) in a rubber plantation area of Kerala, India. Parasitol Res. 2013;112:1299-304.

69. Sumodan PK, Vargas RM, Pothikasikorn J, Sumanrote A, Lefait-Robin R, Dujardin J-P. Rubber plantations as a mosquito box amplification in South and Southeast Asia. In: Morand S, Dujardin J-P, Lefait-Robin R, Apiwathnasorn C, editors. Socio-ecological dimensions of infectious diseases in Southeast Asia. Singapore: Springer; 2015. p. 155-67.

70. Urbanelli S, Bellini R, Carrieri M, Sallicandro P, Celli G. Population structure of Aedes albopictus (Skuse): the mosquito which is colonizing Mediterranean countries. Heredity. 2000;84:331-7.

71. Khambhampati S, Black WC, Rai KS. Geographic origin of the US and Brazilian Aedes albopictus inferred from allozyme analysis. Heredity. 1991;67:85-94.
72. Hawley WA, Reiter P, Copeland RS, Pumpuni CB, Craig GB. Aedes albopictus in North America probable introduction in used tires from northern Asia. Science. 1987;236:1114-6.

73. Urbanski J, Mogi M, O'Donnell D, De Cotiis M, Toma T, Armbruster P. Rapid adaptive evolution of photoperiodic response during invasion and range expansion across a climatic gradient. Am Nat. 2012;179:490-500.

74. Poelchau MF, Reynolds JA, Elsik CG, Denlinger DL, Armbruster PA. Deep sequencing reveals complex mechanisms of diapause preparation in the invasive mosquito, Aedes albopictus. Proc Biol Sci. 2013;280:20130143.

75. Maynard AJ, Ambrose L, Cooper RD, Chow WK, Davis JB, Muzari MO, et al. Tiger on the prowl: invasion history and spatio-temporal genetic structure of the Asian tiger mosquito Aedes albopictus (Skuse, 1894) in the Indo-Pacific. PLoS Negl Trop Dis. 2017;1 1:e0005546.

76. Birungi J, Munstermann LE. Genetic Structure of Aedes albopictus (Diptera: Culicidae) populations based on mitochondrial ND5 sequences: evidence for an independent invasion into Brazil and United States. Ann Entomol Soc Am. 2002;95:125-32.

\section{Publisher's Note}

Springer Nature remains neutral with regard to jurisdictional claims in published maps and institutional affiliations.
Ready to submit your research? Choose BMC and benefit from:

- fast, convenient online submission

- thorough peer review by experienced researchers in your field

- rapid publication on acceptance

- support for research data, including large and complex data types

- gold Open Access which fosters wider collaboration and increased citations

- maximum visibility for your research: over 100M website views per year

At BMC, research is always in progress.

Learn more biomedcentral.com/submissions 